\title{
A New Structural Model for Graphene Oxide and Reduced Graphene Oxide as Revealed by Core EELS and DFT
}

\author{
Anna Tararan ${ }^{1}$, Alberto Zobelli ${ }^{1}$, Ana Benito ${ }^{2}$, Wolfgang Maser $^{2}$, Odile Stephan $^{1}$ \\ 1. Laboratoire de Physique des Solides, Univ. Paris-Sud, CNRS UMR 8502, F-91405, Orsay, France \\ 2. Department of Chemical Processes and Nanotechnology, Instituto de Carboquímica ICB-CSIC, \\ C/Miguel Luesma Castán 4, E-50018 Zaragoza, Spain
}

Graphite oxide (GO) is a well known material since the middle of the XIX century. In the latest years it has attracted a renewed fame and interest as a precursor for a cheap large-scale production of graphene. Indeed, GO conserves the layered structure of graphite with an expanded interlayer distance that facilitates exfoliation, a subsequent reduction yields a material whose properties are very similar to those of graphene. Reduced graphene oxide (RGO) properties strongly depend on the local structure and stoichiometry. However, about 150 years from the first synthesis, many questions remain still open about GO and RGO chemical homogeneity and the functional groups effectively present.

Previous spectroscopy studies stated that the oxygen content in graphene oxide ranges from 22 to $32 \%$. However, transmission electron microscope images revealed that GO is a very inhomogeneous material at the nanometer scale. Still, no spatially resolved spectroscopic studies have yet been reported and only average evaluations are provided in literature. Electron Energy Loss Spectroscopy (EELS) in a Scanning Transmission Electron Microscope (STEM) could give access to the suitable scale but GO and RGO are highly sensitive to irradiation.

In this study we overcame this limitation by adopting an experimental set up combining a liquid nitrogen cooling system at the sample stage, a low accelerated electrons beam $(60 \mathrm{keV})$ and a liquid nitrogen cooled CCD camera with a low read-out noise of three counts r.m.s. and a negligible dark count noise. Hyperspectral core EELS images have been then acquired in a low dose mode (order of 105 $\mathrm{e}-/ \mathrm{nm} 2$ ) at a $10 \mathrm{~nm}$ spatial resolution which guaranties a sufficient signal over noise ratio.

Chemical maps for GO and RGO (see figure) show regions within individual flakes with different oxidation levels confirming the non homogeneity of the material. Whereas oxygen rates averaged over the whole area are in agreement with literature results, we observe that the oxygen content can locally rise up to $60 \%$.

Lower oxidized GO regions present fine structure at the carbon K-edge similar to those of amorphous graphene, while highly oxidized regions present specific core EELS signatures. RGO samples show the well-known fine structure profile typical of graphite, proving an excellent restoration of the carbon network. Nevertheless regions characterized by residual oxygen exhibit an additional sharp peak.

These results have been combined with complementary DFT simulations. An analysis of formation and binding energies for different oxygen functional groups and concentrations and EELS spectra simulations allowed us to provide a new structural model for GO and RGO compatible with our experimental findings. Given the extremely high carbon over oxygen ratio, we suggest for the strongly oxidized regions a structure fully functionalized by hydroxyl groups, while in lower oxidized regions also the contribution from epoxides is expected. 

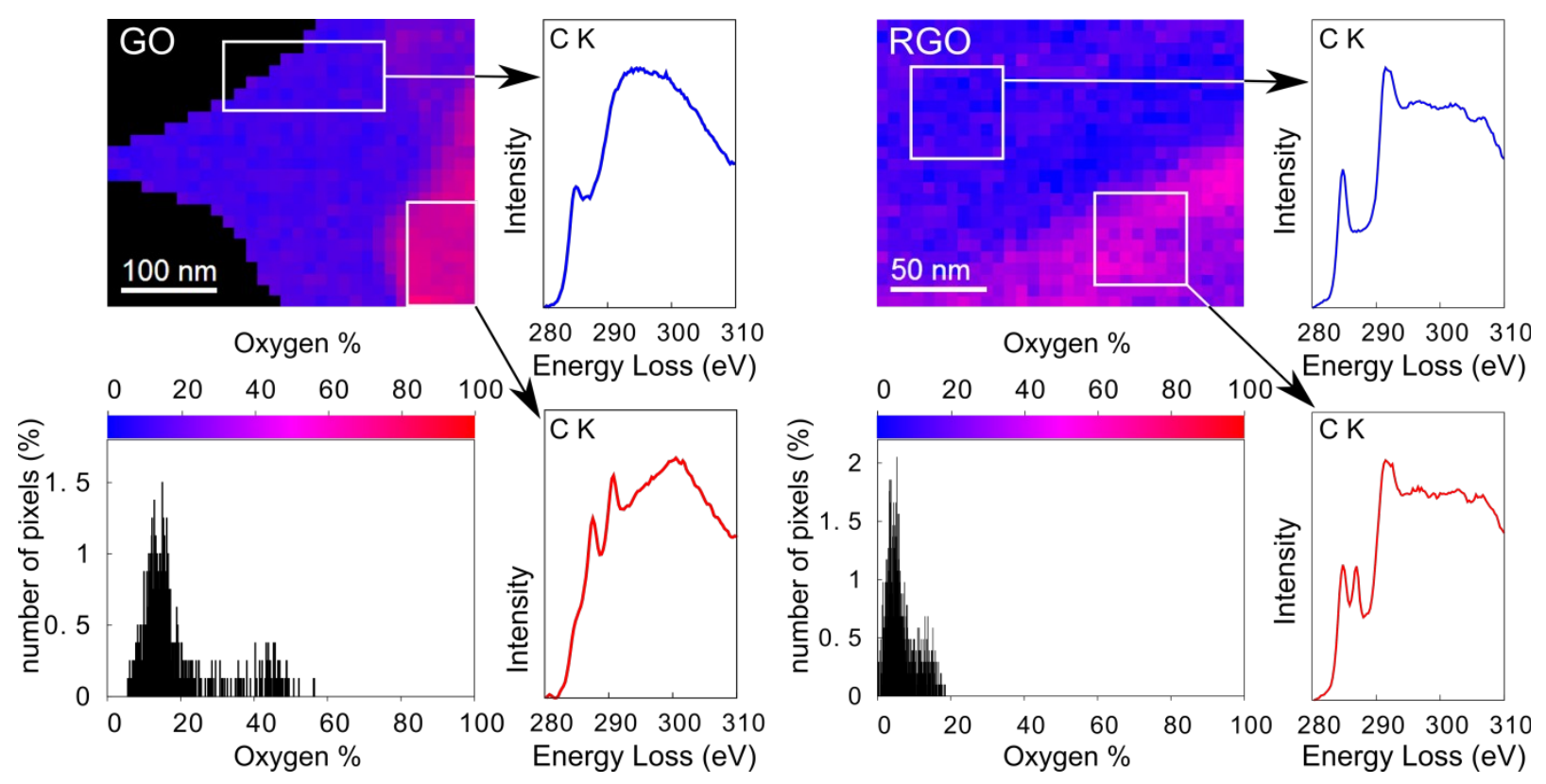

Figure.1 Chemical maps of GO and RGO flakes and related histogram of oxygen occurrence. Carbon K-edges fine structures extracted from the marked regions in the maps. 